\title{
Vortex wake and energy transitions of an oscillating cylinder at low Reynolds number
}

\author{
B. Stewart* J. Leontini* K. Hourigan* \\ M. C. Thompson*
}

(Received 25 October 2004, revised 15 February 2005)

\begin{abstract}
To better understand vortex-induced vibration at low Reynolds number, a two-dimensional numerical study of the forced oscillation of a cylinder in cross-flow was undertaken. The Kármán vortex shedding mode $(2 S)$ of the forced oscillation of a cylinder in cross-flow incorporated a gradual change from positive to negative energy transfer with increasing amplitude of motion. As amplitude was increased, the $2 S$ shedding mode evolved into an asymmetric mode of shedding in which a pair and a single vortex $(P+S)$ were shed each motion cycle. Energy contours were established in the region of primary lock-in and the boundary of zero energy transfer was defined. The observed $P+S$ mode only occurred in the region of negative energy transfer.
\end{abstract}

*Fluids Laboratory for Aeronautical and Industrial Research, Dept. Mechanical Engineering, Monash University, Melbourne, Australia.

mailto:kerry . hourigan@eng. monash. edu . au

See http://anziamj.austms.org.au/V46/CTAC2004/Stew for this article, (c) Austral. Mathematical Soc. 2005. Published April 22, 2005. ISSN 1446-8735 


\section{Contents}

1 Introduction

C182

2 Computational Methodology C184

2.1 Numerical Code . . . . . . . . . . . . . . . . . . C185

3 Results

C187

4 Conclusions

C193

References

C194

\section{Introduction}

A common approach to understanding vortex-induced vibration (vIV) has been to investigate the near wake and energy transfer characteristics of a bluff body undergoing forced cross-flow oscillation, see reviews in [10] and [12]. This approach provides an indication of flow regimes that may produce VIV and the resulting amplitudes of oscillation. However, little fundamental research has been conducted to investigate the energy transfers present at Reynolds number $\operatorname{Re}<500$.

The current numerical investigation aims to define the region of positive energy transfer for a cylinder undergoing forced oscillation transverse to the flow at $R e=200$. Experimentation has found that forcing a cylinder to oscillate transverse to a flow may cause the vortex shedding of the cylinder to synchronize with the imposed motion along its entire length [7]. In this way, oscillations near the natural shedding frequency $f_{n}$ may extend the laminar flow range from $\mathrm{Re}=150$ to approximately $350[4,7]$. This results in physical flows that may be accurately approximated by two-dimensional simulations. 
As vortices are shed from a structure, vortex formation takes place from the interaction of four regions of vorticity generated at the body during each period of motion $[2,13]$. These regions may then interact to shed two, three or four vortices into the wake each cycle. For oscillations near the fundamental lock-in region, a wake mode shedding two pairs of opposite sign vortices $(2 P)$ has been observed [13], but for flows with $\operatorname{Re}<300$ this mode is replaced by a pair and a single vortex $(P+S)$ being shed $[2,5,7]$.

Carberry et al. [3] found experimentally that changes in the lift force, and hence the energy transfer, of a cylinder undergoing forced oscillation was intrinsically linked to the formation of the near wake. When traversing the parameter space defined by frequency and amplitude of the body motion, a discontinuity in the phase between the lift force and cylinder displacement was perceived. This discontinuity was commented upon by Bishop and Hassan [1] and has since been observed at frequencies both above and below the natural shedding frequency at a range of Reynolds numbers $[2,6,8]$.

A jump in the phase between lift and displacement is often associated with a change in sign of energy transfer or shedding mode. When the phase between cylinder displacement and lift force was observed to jump through $180^{\circ}$, the direction of energy transfer changed from positive to negative or vice versa $[1,2,3,9,13]$. Hover et al. [6] reproduced contours of energy transfer from data at $\mathrm{Re}=3800$ and 10000 in the non-dimensional amplitude-frequency plane; they found that the contour of zero energy transfer agreed reasonably with the data obtained from free vibration of the cylinder at $\operatorname{Re}=3800$. This result indicated that analysis of forced vibration might be a useful tool for predicting the regions in which vortex-induced vibration is possible. Additionally this type of analysis can provide an upper limit for the amplitude of oscillation occurring in VIV. In conjunction with this type of study an examination of the wake at various amplitudes and frequencies of oscillation may be conducted in order to determine a mechanism in the near wake having a direct influence on the direction of energy transfer. 


\section{Computational Methodology}

The cylinder motion

$$
y(t)=A \cos \left(2 \pi f_{o} t\right),
$$

where $A$ is the amplitude of displacement and $f_{o}$ is the forcing frequency. Results are described in terms of the scaled amplitude $A / D$, and the reduced velocity

$$
V_{r}=\frac{U}{f_{o} D},
$$

which is equivalent to the wavelength of the cylinder motion scaled by the cylinder diameter $D$. Attention was focused on the primary lock-in region of the parameter space. Throughout the investigation, the scaled amplitude was varied between 0.1 and 1.0 and the reduced velocity between 1.0 and 8.0. No fewer than 80 data sets were collected at irregularly spaced intervals throughout this region. The cylinder was constrained to move transverse to the upstream flow and hence the drag force played no part in the energy transfer. Analysis of the drag force was therefore neglected throughout this study.

Also used to characterise the wake was the Strouhal frequency

$$
\mathrm{St}=\frac{f_{n} D}{U} .
$$

Data analysis was carried out using the lift coefficient $C_{L}$ and non-dimensional energy transfer per motion cycle

$$
E=-\frac{1}{D} \int_{0}^{T} A \sin \left(2 \pi f_{o} t\right) C_{L}(t) d t
$$

as defined by [2]. $E$ indicates the component of $C_{L}$ in phase with the cylinder velocity over one period of motion $T$ and positive $E$ indicates work done by the fluid on the cylinder. The energy integral given by equation (4) was evaluated using a composite Simpson's Rule. For consistency, the time was scaled by $U / D$, giving dimensionless time $\tau$. 


\subsection{Numerical Code}

The two-dimensional numerical code utilized a spectral-element scheme with three-step time splitting to solve the incompressible Navier-Stokes equations with an additional forcing term. Eighth-order Lagrangian interpolating polynomials were used to approximate the solution variables within the macroelements of the mesh, see [11] for more details. The mesh (shown in Figure 1) consisted of quadrilateral elements with the domain extending $23 D$ downstream and $15 D$ upstream and to each of the transverse boundaries. The mesh was attached to the non-inertial frame of reference of the cylinder and the free-stream flow and cylinder motion were started impulsively from rest. A no-slip condition was enforced at the cylinder body.

Detailed resolution and convergence tests have previously been conducted for this system at a variety of Reynolds numbers and were found to give results that compared well with accepted values [11]. For completeness, a time-step analysis was carried out for the fixed and forced cylinders in the current investigation. Time-steps of $\Delta \tau=0.001$ and 0.002 were used to check convergence of the solution. In the case of the fixed cylinder, the smaller time-step led to a slightly faster flow development but the vortex shedding frequency for the two solutions was found to be identical to 5 significant figures over any defined time interval. In the case of the cylinder undergoing forced oscillations, the flow development was dictated by the cylinder motion and the lift and drag coefficients were within $0.1 \%$ after $\tau=200$. Consequently $\Delta \tau=0.001$ was deemed to give sufficient accuracy and was used in all subsequent simulations. A fixed cylinder simulation run at $\mathrm{Re}=200$ for $\tau>350$ provided the natural shedding frequency of the cylinder. Using an FFT over the entire lift force data series, equation (3) gave $\mathrm{St}=0.198$. 


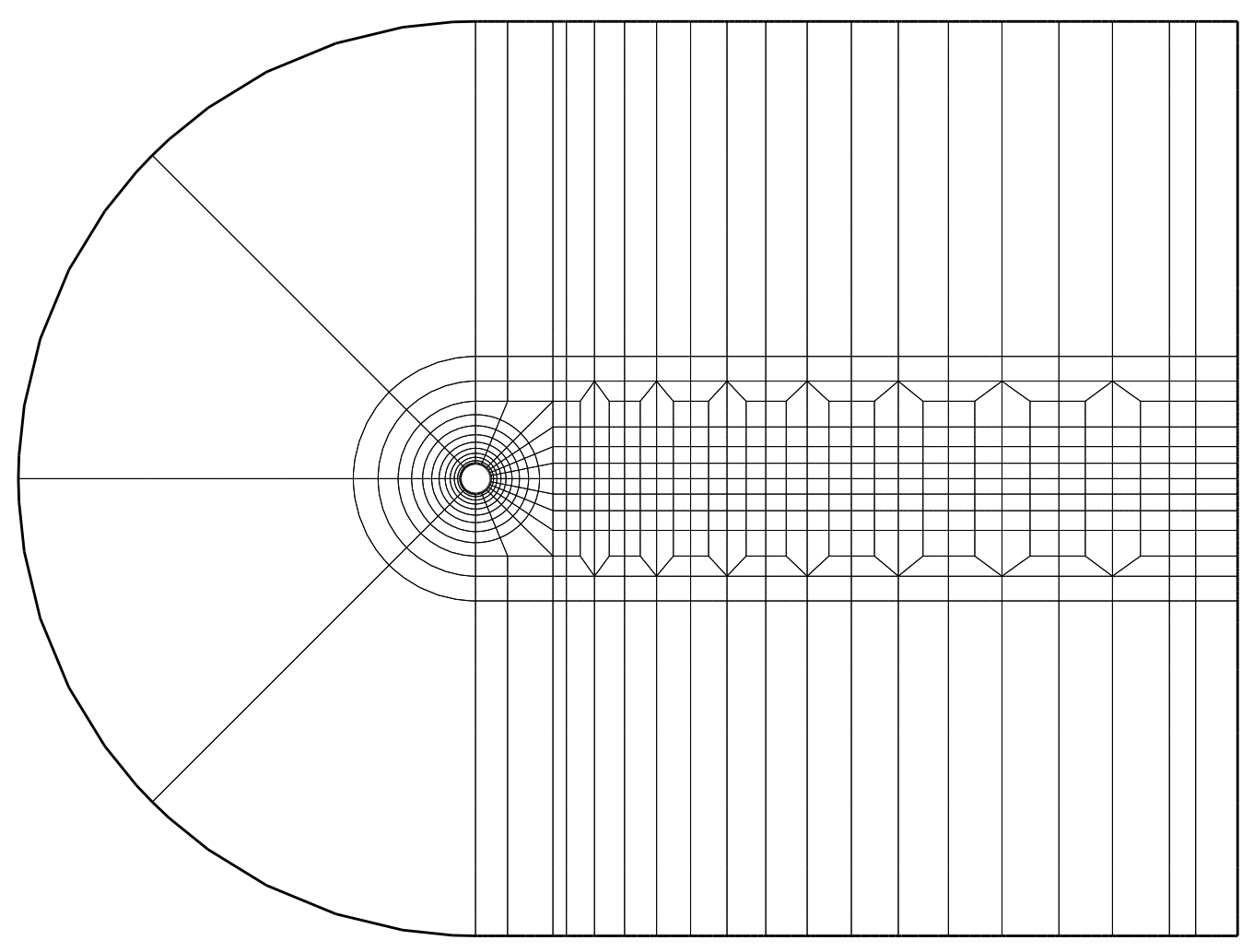

FiguRE 1: Macro-element mesh used during investigation. 


\section{$3 \quad$ Results}

The wake was considered synchronized with the cylinder motion when a plot of cylinder displacement versus lift traced out a steady, closed loop [2] and the energy transfer per cycle had reached a constant value. For certain forcing frequencies near $f_{n}$, synchronization was difficult to obtain, with a small variation in the frequency altering the lift data from a steady synchronized form to a slowly varying series with a small constant change in $E$ each cycle. This behaviour continued for $t>500$ with no sign of stabilising. Such sensitivity of cylinder wakes near the Strouhal frequency has been observed in previous numerical investigations $[2,9]$.

At reduced velocities below 4.5, wake visualisations often showed an apparently stable Kármán wake; however, a prominent beating was present between $f_{o}$ and $f_{n}$. Although the forcing frequency was dominant, the interaction of these two frequencies led to a beating in the form of the energy transfer per motion cycle and E consequently varied about a mean value. The varying nature of the energy transfer led this region to be classified as non-synchronised. Areas in which this nature was particularly apparent were at $3 f_{n}$ and $3 / 2 f_{n}$. Near these frequencies, the energy transfer oscillated about a mean value that was becoming increasingly negative. Figure 2(a) shows a wake in this region for which the interaction between $f_{n}$ and $f_{o}$ was clearly apparent. The low frequency modulation produced by $f_{n}$ caused the timing of vortex shedding to vary slightly each period resulting in the wake shown.

As $V_{r}$ increased to values near 4.5 , the vortex shedding locked on. This occurred first at amplitudes above 0.5 where the effects of cylinder motion were more pronounced. As $f_{o}$ approached $f_{n}$ synchronisation occurred at all values of $A / D$. This lock-on was observed throughout the parameter space until $V_{r}>6.5$, at which point the wake pattern started to become disordered. This boundary coincided closely with that established by [13], defining the limits of the fundamental synchronization region. 
(a)

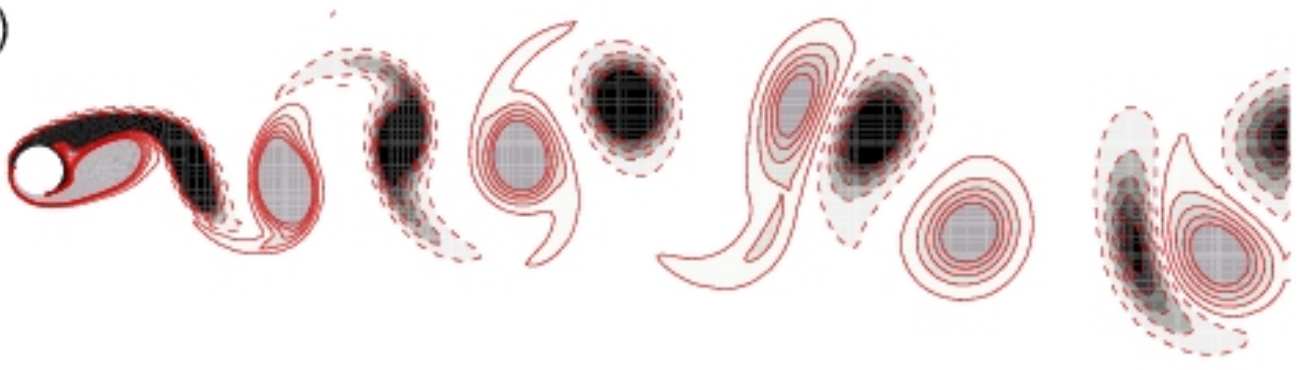

(b)

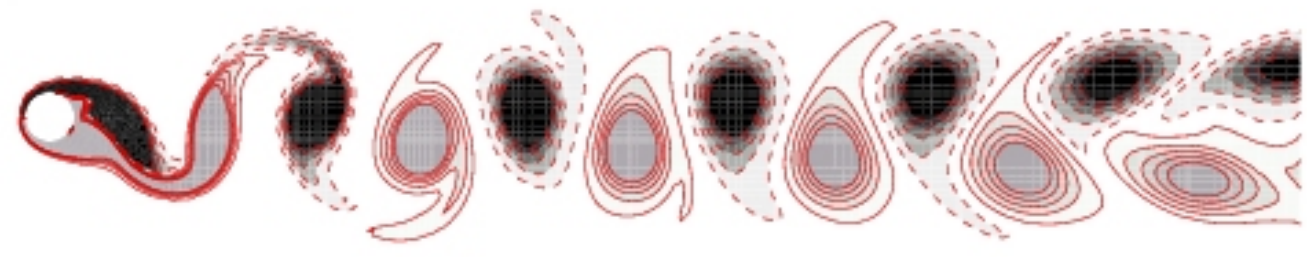

(c)

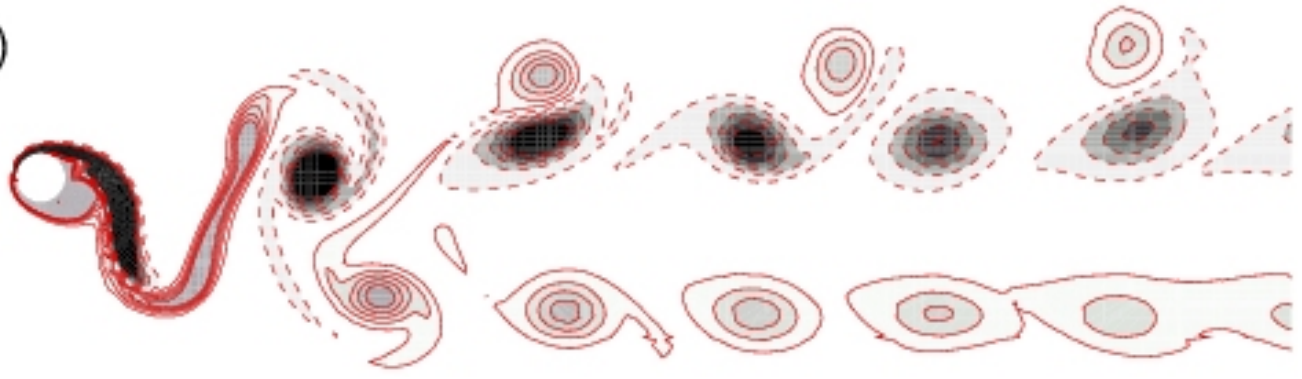

FigURE 2: Vorticity contours for (a) high frequency unstable wake at $V_{r}=3$, $A=0.179 D$ showing the low frequency modulation, (b) synchronised $2 S$ shedding at $V_{r}=5, A=0.199 D$ and (c) synchronised $P+S$ shedding at $V_{r}=5, A=0.696 D$. Negative vorticity is shown in black and enclosed by dashed contours. 
As the amplitude of oscillation was increased from a value of 0.2 in the synchronization region, the lift trace varied continuously from a sinusoidal trace, out of phase with the cylinder displacement, to an asymmetric mode becoming apparent at values of $A / D>0.6$. This represented the progression from the standard $2 S$ or Kármán street wake, such as that shown in Figure 2(b), to the $P+S$ mode of shedding. $2 S$ shedding occurred at low amplitudes and displayed a gradual change from positive to negative energy transfer with increasing amplitude. $P+S$ shedding was observed only in the region of negative energy transfer. Figure 2(c) shows a typical $P+S$ shedding, with asymmetric pairing of a positive and negative vortex along the top of the wake, with the outermost vortex being rapidly convected downstream.

To illustrate better the transition from positive to negative energy transfer, lift data are shown along with cylinder displacement for $V_{r}=5.0$ at three different amplitudes within the lock-on region (Figures 3(a), (b) and (c)).

It was apparent that although the lift phase experienced a shift between the $2 S$ shedding in Figure 3(a) and the $P+S$ shedding in Figure 3(c), the wake mechanism governing this shift was not obvious. During the $2 S$ shedding, the lift progressed from a near sinusoidal trace to one that contained two smaller, secondary peaks per oscillation, shown in Figure 3(b)i. These peaks became apparent at amplitudes between 0.40 and 0.45 in the primary lock-on region. The flow remained symmetric with secondary maxima and minima developing in the lift trace each cycle. Following the development of the secondary peaks, an increase in the amplitude caused the major peaks and troughs to reduce until all were of approximately equal magnitude.

Further increases in $A / D$ caused a growth in alternate peaks, creating a phase shift between lift and displacement. At this time the wake was still symmetric and shedding in the $2 S$ mode. An increase in amplitude following this switch led to the disappearance of one of the remaining secondary peaks (Figure 3(c)i)) and the development of the asymmetric, $P+S$ mode mentioned previously. One such peak transition occurred at the time indicated by dots in Figures 3(a)i), (b)i) and (c)i) at which wake images were obtained. 

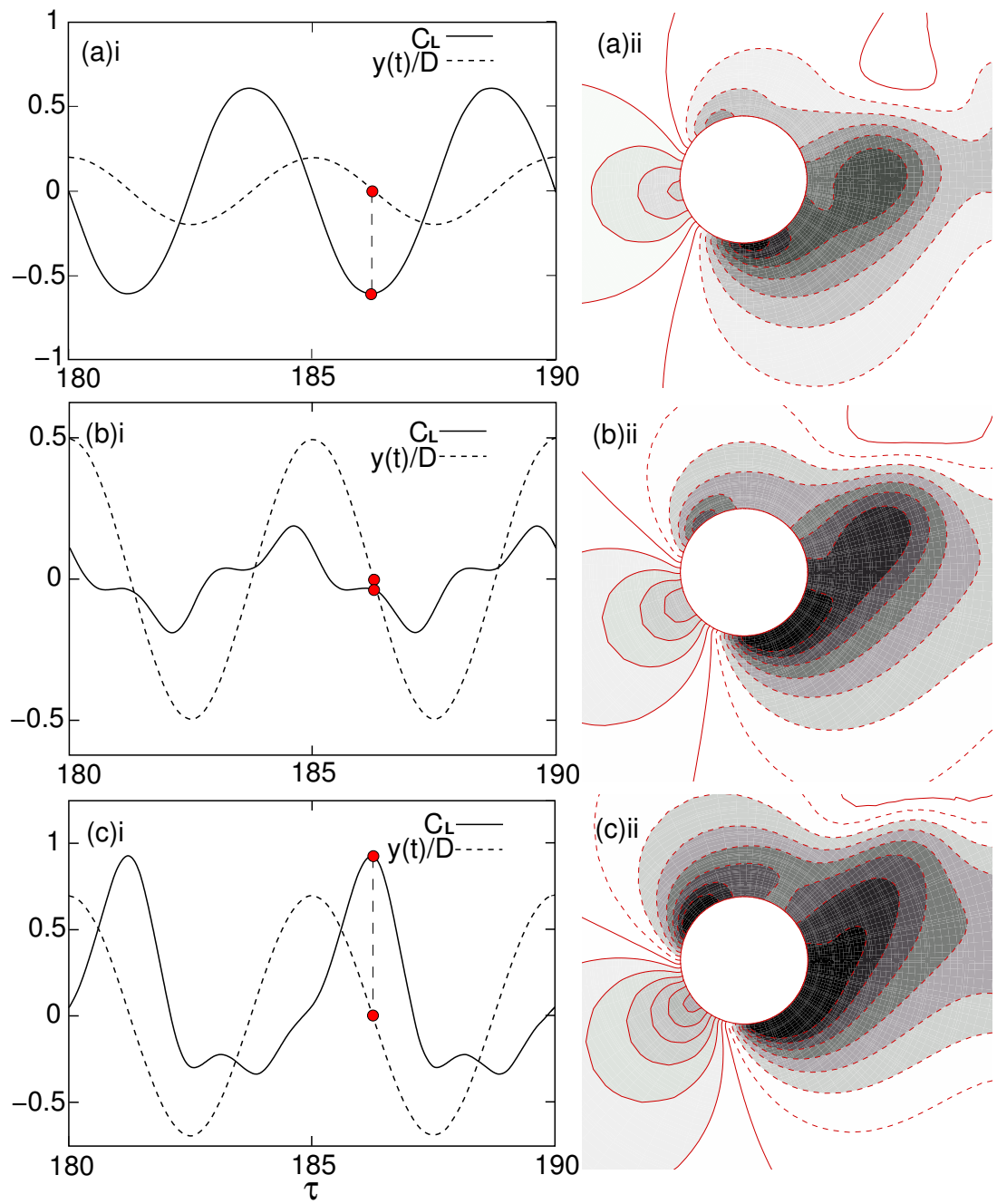

FiguRE 3: Lift coefficients (i) and corresponding pressure contours (ii) for cylinder oscillating at $V_{r}=5.0$, (a) $A / D=0.199$, (b) $A / D=0.497$ and (c) $A / D=0.696$. Dots indicate point at which images were obtained. 
The phase shift corresponded to a change in the direction of energy transfer. Although the switch occurred as the result of a continuous process, the transition took place over a small amplitude range and was completed within a variation of $A / D<0.1$. At the time that images were obtained, the cylinder was at maximum downward velocity and the lift coefficient changed dramatically from an extreme negative in Figure 3(a)i to almost zero in Figure 3(b)i and an extreme positive in Figure 3(c)i.

Three dominant pressure regions affected the lift force on the cylinder. These were the high pressure area at the front stagnation point and the low pressure regions corresponding to the top and bottom shear layers, occurring just before separation. In the pressure contours of Figures 3(a)ii, (b)ii and (c)ii, the negative pressure in the shear layers is shown in black and enclosed by dashed lines. As the amplitude was increased, these regions of positive and negative pressure were displaced further around the cylinder during maximum velocity. This shift made a substantial difference in the overall lift when the pressures from the shear layers were nearly equal.

In Figure 3(a)ii, the cylinder's downward motion caused acceleration of the lower shear layer which created an area of low pressure near the bottom of the cylinder and a net downward lift force $\left(C_{L}\right.$ negative). This differed from the wake structure at amplitudes near 0.5 (Figure 3(b)ii)), when the front stagnation point was shifted towards the bottom of the cylinder. This high pressure region partially offset the low pressure created in the lower shear layer, resulting in a near zero value for $C_{L}$.

At amplitudes in the region of 0.7 , the $P+S$ mode of shedding occurred and the elongated region of positive vorticity shedding from the bottom of the cylinder separated into two distinct vortices downstream. The elongation of the lower shear structure moved the concentration of vorticity further from the cylinder and the low pressure in the upper shear layer dominated, giving a maximum positive $C_{L}$. During the upwards motion of the cylinder in this asymmetric wake mode, negative vorticity from the upper shear layer formed a low pressure region much closer to the back of the cylinder. This 


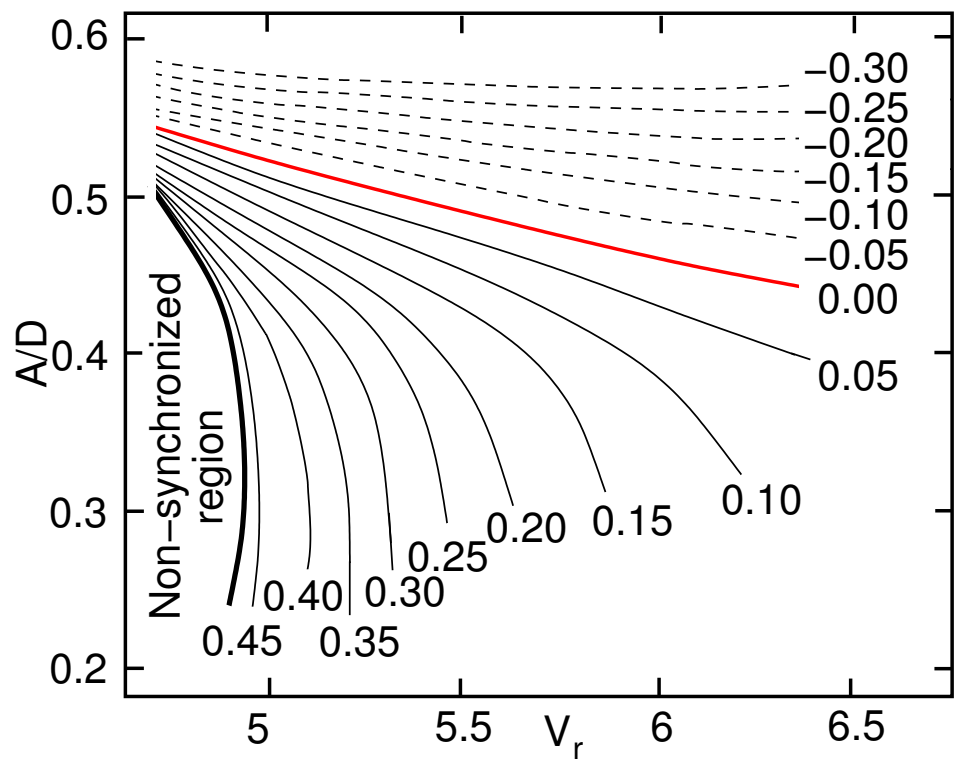

FiguRE 4: Contours of constant energy transfer in the primary lock-in region. Values of $E$ are shown and negative energy transfer is indicated with dashed lines.

contributed a larger component to the upward lift, resulting in the net downward lift being of much lower amplitude than the net upward lift generated in the previous half cycle.

From energy measurements throughout the lock-in region, contours of energy transfer per cycle were established (Figure 4). These contours of energy $E$ indicated that the energy transition took place between amplitudes of 0.45 and 0.55 . For VIV to occur, a positive energy transfer is required to account for losses due to structural damping [3,6], hence this boundary represents an upper limit to the observed oscillation amplitude.

The left-hand boundary of Figure 4 represents the transition to the nonsynchronised mode of shedding mentioned earlier. Across this boundary, 
vortex lock-on was observed to occur suddenly, with an associated change in the vortex shedding. With the onset of synchronization, the shedding shear layer switched from one side of the cylinder to the other. This switch also incorporated a change from a time varying energy transfer, with an average negative $E$, to a steady positive $E$. This suggests some mechanism linking the energy transfer and shedding mode at the synchronization boundaries. At amplitudes above the energy transition, values of $E$ became steadily more negative and were dependent primarily on the amplitude, rather than the reduced velocity. This may be linked to the findings of [9] where the $P+S$ wake developed at almost constant amplitude.

\section{Conclusions}

A numerical investigation of two-dimensional flow past an oscillating cylinder at $R e=200$ obtained data for the lift force and wake mode occurring at various frequencies and amplitudes of motion. The region in which vortex shedding synchronized with the cylinder motion was determined and energy transfer calculated for all points within this region. Following analysis of the wake modes, it was discovered that the $2 S$ shedding mode displayed a gradual decrease in energy transfer as the reduced velocity and amplitude increased. This was brought about by the development of two secondary peaks in the lift force. At a critical amplitude, these peaks switched dominance, affecting a shift in phase between lift and displacement, and a change in direction of energy transfer. This change in energy was closely related to the position of the stagnation region during the motion cycle. A further increase in amplitude, following the energy transition saw the onset of asymmetric $P+S$ shedding in regions of negative energy transfer and amplitudes above 0.6. A plot of energy contours (Figure 4) indicated that an energy transition took place over a fairly small range of amplitudes in the primary lock-on region. This result indicated the occurrence of maximum VIV amplitudes of between 0.45 and 0.55 at reduced velocities between 4.5 and 6.5. 


\section{References}

[1] R. E. D. Bishop and A. Y. Hassan. The lift and drag forces on a circular cylinder oscillating in a flowing fluid. Proc. R. Soc. Lond. A, 277: 51-75, 1964. C183

[2] H. M. Blackburn and R. D. Henderson. A study of two-dimensional flow past an oscillating cylinder. J. Fluid Mech., 385: 255-286, 1999. C183, C184, C187

[3] J. Carberry, J. Sheridan and D. Rockwell. Forces and wake modes of an oscillating cylinder. J. Fluids Struct., 15: 523-532, 2001. C183, C192

[4] O. M. Griffin. The unsteady wake of an oscillating cylinder at low Reynolds number. J. Appl. Mech., 38: 729-738, 1971. C182

[5] O. M. Griffin and S. E. Ramberg. The vortex-street wakes of vibrating cylinders. J. Fluid Mech., 66: 553-576, 1974. C183

[6] F. S. Hover, A. H. Techet and M. S. Triantafyllou. Forces on uniform and tapered cylinders in crossflow. J. Fluid Mech., 363: 97-114, 1998. C183, C192

[7] G. H. Koopmann. The vortex wakes of vibrating cylinders at low Reynolds numbers. J. Fluid Mech., 28: 501-512, 1967. C182, C183

[8] X.-Y. Lu and C. Dalton. Calculation of the timing of vortex formation from an oscillating cylinder. J. Fluids Struct., 10: 527-541, 1996. C183

[9] J. R. Meneghini and P. W. Bearman. Numerical simulation of high amplitude oscillatory flow about a circular cylinder. J. Fluids Struct., 9: 435-455, 1995. C183, C187, C193 
[10] T. Sarpakaya. A critical review of the intrinsic nature of vortex-induced vibrations. J. Fluids Struct., 19: 389-447, 2004. C182

[11] M. C. Thompson, K. Hourigan and J. Sheridan. Three-dimensional instabilities in the wake of a circular cylinder. Exp. Therm. Fluid Sci., 12: 190-196, 1996. C185

[12] C. H. K. Williamson and R. Govardhan. Vortex-induced vibrations. Ann. Rev. Fluid Mech., 36: 413-455, 2004. C182

[13] C. H. K. Williamson and A. Roshko. Vortex formation in the wake of an oscillating cylinder. J. Fluids Struct., 2: 355-381, 1988. C183, C187 